\title{
Searches for a doubly charged scalar at LHC and future colliders
}

\section{Margherita Ghezzi*}

Paul Scherrer Institut, Switzerland

E-mail: margherita.ghezzi@psi.ch

Doubly charged scalars are part of the spectrum of various Beyond-the-Standard-Model scenarios, from mechanisms for the generation of the neutrino masses to the Left-Right models. Direct searches in the pair production channel have been performed at the LHC, setting bounds on the mass of the particle. Future linear colliders will have a great potential to test the direct production of this particle as well as its couplings to the charged leptons. Moreover, as this particle allows for lepton flavour violation, complementary bounds are also provided by low energy measurements.

An Alpine LHC Physics Summit (ALPS2018)

15-20 April, 2018

Obergurgl, Austria

${ }^{*}$ Speaker. 


\section{Introduction}

Doubly charged scalars (DCSs) are hypothetical Beyond-the Standard-Model (BSM) particles with a long history in literature. They first appeared in the context of Left-Right models [1-3]. They are also present in many BSM extensions for low-energy observables [4-7] and in BSM scenarios motivated by mechanisms for the neutrino mass generation [8-14]. In the following we will consider a minimal scenario obtained by adding to the SM field content only a $S U(2)_{L}$-singlet DCS that couples to the right-handed fermions of the lepton sector

$$
\begin{aligned}
\mathscr{L}_{\mathrm{UV}} & =\mathscr{L}_{\mathrm{SM}}+\left(D_{\mu} S^{++}\right)^{\dagger}\left(D^{\mu} S^{++}\right)+\left(\lambda_{a b}{\overline{\left(\ell_{R}\right)_{a}}}^{c}\left(\ell_{R}\right)_{b} S^{++}+\text {h.c. }\right)+ \\
& +\lambda_{2}\left(H^{\dagger} H\right)\left(S^{--} S^{++}\right)+\lambda_{4}\left(S^{--} S^{++}\right)^{2}+[\ldots],
\end{aligned}
$$

where $a$ and $b$ are flavour indices and $\lambda_{a b}$ is a complex coupling matrix in the flavour space. The scalar potential introduces also a coupling to the Higgs sector, controlled by $\lambda_{2}$, and a quartic selfinteraction of the DCS, controlled by the parameter $\lambda_{4}$. As no specific assumption on the origin of $m_{S}$ is made, $\lambda_{2}$ and the DCS mass $m_{S}$ are understood to be unconstrained by the electroweaksymmetry-breaking mechanism. Any further form of new physics contributing to the value of $m_{S}$ and of the DCS width $\Gamma_{S}$ is intended to be represented by the ellipsis. The phenomenology of the DCS in the Higgs sector can be completely separated from the the phenomenology in the leptonic sector, by means of the independent parameter $\lambda_{2}$. In the following the focus will be on the effects of the DCS in the leptonic sector. In particular, some of the phenomenological results presented in [15] will be reviewed.

The DCS described by Eq. 1.1 breaks explicitly the lepton number and the lepton flavour conservation. Therefore, its phenomenology is strongly connected with the measurements of charged LFV processes at low-energy facilities. The current experimental limits are reported in Table 1. DCSs contribute at tree level to three-body decays of charged leptons: the limits on the branching ratios for such decays are listed in the left column of the table. Moreover, DCSs contribute to LFV decays at the one-loop level: the limits of these processes are given in the right column of the table, together with the limit on $\mu \rightarrow e$ conversion. The latter receives contributions from the DCS through the effective operators involving quarks generated by the QED renormalization group evolution. Finally, the DCS also generates muonium-antimuonium oscillations at tree level, whose experimental bound is $\mathscr{P}(\bar{M}-M)=8.3 \times 10^{-11} / S_{B}$, with $S_{B}=0.35$ in case of interactions consisting of right-handed currents [16]. For most of the processes mentioned above there are planned experiments that are going to increase the sensitivity by one or more orders of magnitude [17-20].

\section{Direct searches at the LHC}

ATLAS and CMS have performed dedicated searches of the DCS with the $7 \mathrm{TeV}$ and the $13 \mathrm{TeV}$ LHC data sets [26-29]. The ATLAS search [28] has been performed with $36.1 \mathrm{fb}^{-1}$ of integrated luminosity at $13 \mathrm{TeV}$ and distinguishes between two cases: a DCS coupling to left-handed leptons and a DCS coupling to right-handed leptons. The expected signature is two pairs of same-sign leptons at high invariant mass, produced by the decay of a DCS pair. Lower bounds on the mass of the DCS have been set, ranging from $320 \mathrm{GeV}$ to $870 \mathrm{GeV}$ depending on the left/right case and on 


\begin{tabular}{ll|ll}
\hline $\operatorname{Br}\left[\mu^{\mp} \rightarrow e^{\mp} e^{ \pm} e^{\mp}\right] \leq 1.0 \times 10^{-12}$ & {$[21]$} & $\operatorname{Br}[\mu \rightarrow e \gamma] \leq 4.2 \times 10^{-13}$ & {$[22]$} \\
$\operatorname{Br}\left[\tau^{\mp} \rightarrow \mu^{\mp} \mu^{ \pm} \mu^{\mp}\right] \leq 1.2 \times 10^{-8}$ & {$[23]$} & $\operatorname{Br}[\tau \rightarrow \mu \gamma] \leq 4.4 \times 10^{-8}$ & {$[24]$} \\
$\operatorname{Br}\left[\tau^{\mp} \rightarrow e^{\mp} e^{ \pm} e^{\mp}\right] \leq 1.4 \times 10^{-8}$ & {$[23]$} & $\operatorname{Br}[\tau \rightarrow e \gamma] \leq 3.3 \times 10^{-8}$ & [24] \\
$\operatorname{Br}\left[\tau^{\mp} \rightarrow e^{\mp} \mu^{ \pm} \mu^{\mp}\right] \leq 1.6 \times 10^{-8}$ & {$[23]$} & \\
$\operatorname{Br}\left[\tau^{\mp} \rightarrow \mu^{\mp} e^{ \pm} \mu^{\mp}\right] \leq 9.8 \times 10^{-9}$ & {$[23]$} & $\operatorname{Br}_{\mu \rightarrow e}^{\mathrm{Au}} \leq 7 \times 10^{-13}$ \\
$\operatorname{Br}\left[\tau^{\mp} \rightarrow \mu^{\mp} e^{ \pm} e^{\mp}\right] \leq 1.1 \times 10^{-8}$ & {$[23]$} & \\
$\operatorname{Br}\left[\tau^{\mp} \rightarrow e^{\mp} \mu^{ \pm} e^{\mp}\right] \leq 8.4 \times 10^{-9}$ & {$[23]$} & \\
\hline
\end{tabular}

Table 1: Current experimental limits on charged LFV processes.

the assumptions on the decay modes of the DCS. Similar lower limits on the DCS mass have been set by the CMS search [29], performed with $12.9 \mathrm{fb}^{-1}$ of integrated luminosity of the $13 \mathrm{TeV}$ data set. This search focuses on a DCS that appears as a component of a $S U(2)_{L}$ triplet. Therefore, it is associated to a singly charged scalar and a neutral scalar, belonging to the same multiplet. The analysis considers both the DCS pair production and the associated production of a DCS and a singly charged scalar, analysing the two channels separately and in combination.

These experimental results have been obtained in the hypothesis of a narrow DCS width and considering only quark-initiated (Drell-Yan) production topologies. In Ref. [15] a recasting of the CMS search [29] at $13 \mathrm{TeV}$ has been performed, including also photon-initiated topologies beside the Drell-Yan topologies, and studying the effects of a large width. Examples of representative topologies are shown in Figure 1. In Figure. 2 the exclusion and discovery reaches are reported for different values of the mass of the DCS and different luminosities, up to $3000 \mathrm{fb}^{-1}$ expected in the high-luminosity phase of the LHC. The significance $\Sigma \equiv S / \sqrt{S+B+(\Delta B)^{2}}$ is set to $\Sigma=2$ for the exclusion and $\Sigma=5$ for the discovery reach. In order to show the width effect the curves relative to the two illustrative cases $\Gamma_{S} / m_{S}=0.01 \%, 20 \%$ are reported. The three panels correspond to the Drell-Yan channel $q \bar{q} \rightarrow e^{+} e^{+} e^{-} e^{-}$, the photon-initiated channel $\gamma \gamma \rightarrow e^{+} e^{+} e^{-} e^{-}$and the whole process $p p \rightarrow e^{+} e^{+} e^{-} e^{-}$.

\section{Perspectives at future leptonic colliders}

Future electron-positron colliders will have a great potential to probe the leptonic sector of BSM models. In particular, advanced operational stages of the International Linear Collider (ILC) [30, 31] and the Compact Linear Collider (CLIC) $[32,33]$ can play a central role both in indirect and direct searches, superseeding the production threshold limits set by the high-luminosity phase of the LHC. In Table 2 the centre-of-mass energies and integrated luminosities expected at ILC and CLIC are reported, according to the ILC Technical Design Report [30] and the CLIC Conceptual Design Report [32].

In this section we review some phenomenological results, presented in Ref. [15], on the discovery potential of these future prototypes in the context of the DCS described by Eq. 1.1. The numerical simulations have been performed with CalcHEP [34], that provides options to automatically take into account the Initial State Radiation and the Beamstrahlung effects, determined by the 


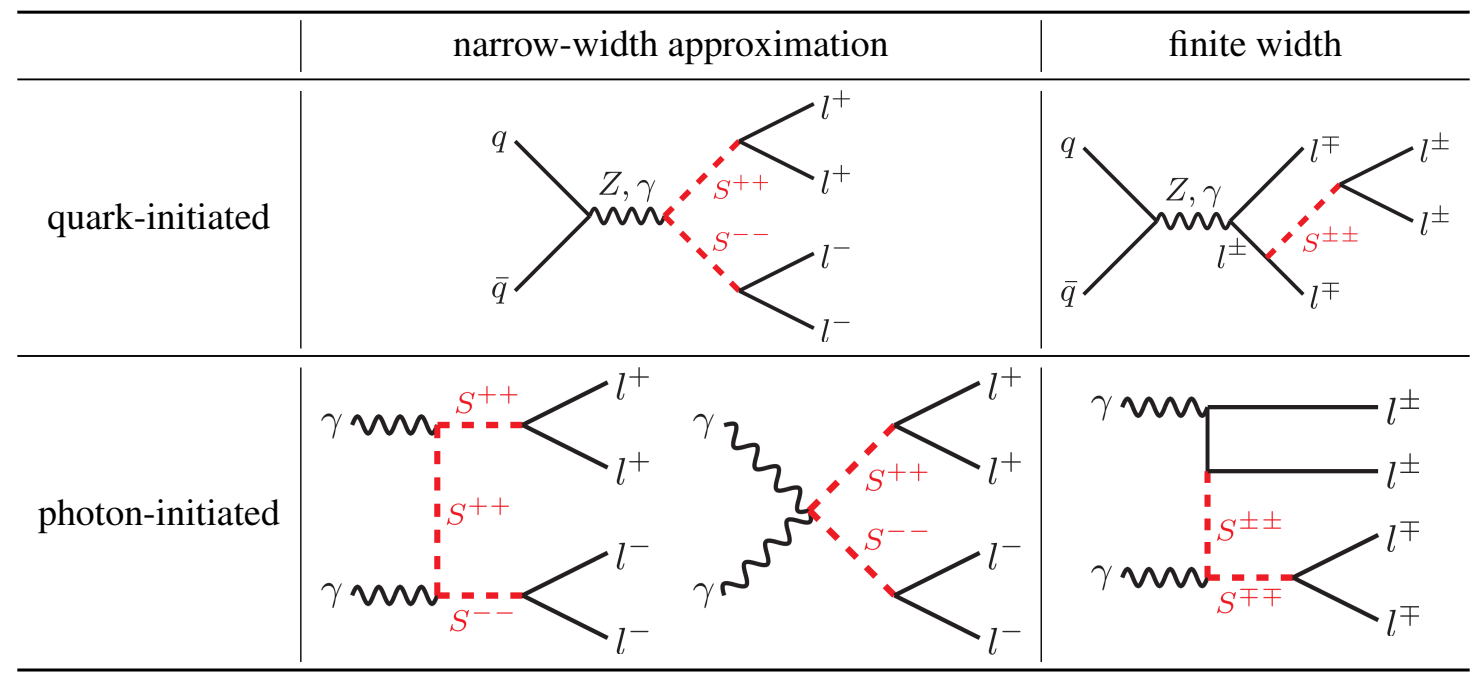

Figure 1: Representative topologies for the process $p p \rightarrow 2 l^{+} 2 l^{-}$, quark-initiated and photoninitiated. The topologies in the last column are neglected in the narrow width approximation but can become relatively important if the DCS width is large.
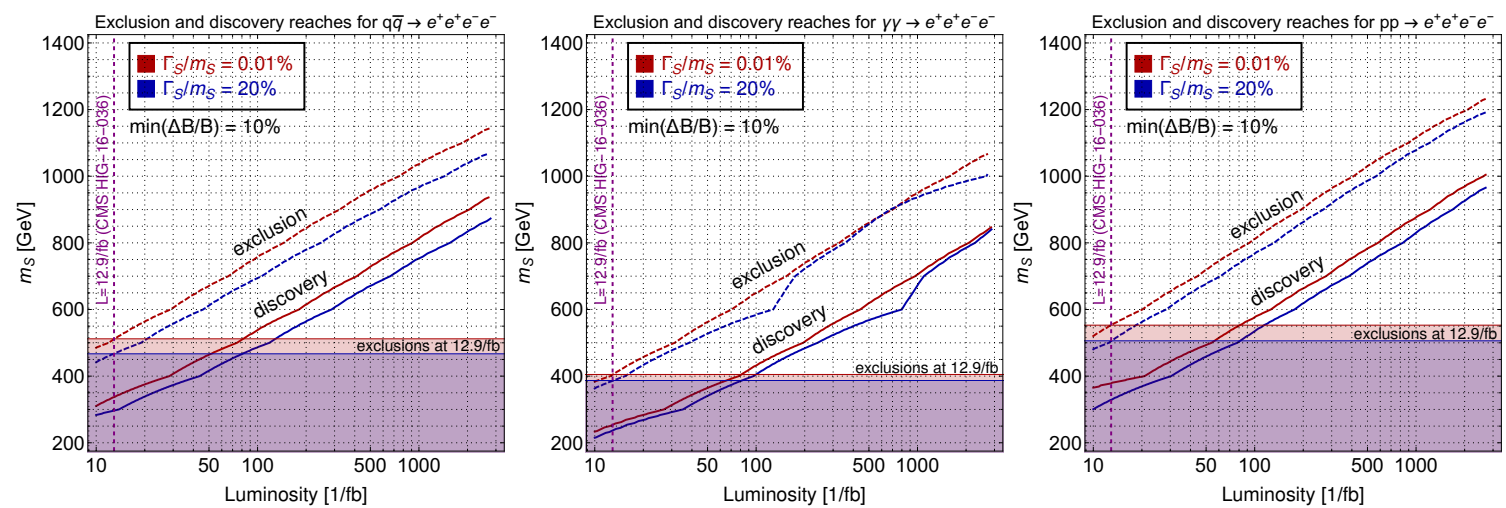

Figure 2: Exclusion and discovery reaches as function of the integrated luminosity. From left to right: quark-initiated process, photon-initiated process and total contribution.

\begin{tabular}{|c|c|c|c|c|c|c|c|c|}
\hline$\sqrt{s}_{\text {ILC }}$ & $250 \mathrm{GeV}$ & $500 \mathrm{GeV}$ & $1 \mathrm{TeV}$ & $\sqrt{s}_{\text {CLIC }}$ & $350 \mathrm{GeV}$ & $380 \mathrm{GeV}$ & $1.5 \mathrm{TeV}$ & $3 \mathrm{TeV}$ \\
\hline $\mathscr{L}_{\text {ILC }}$ & $250 \mathrm{fb}^{-1}$ & $500 \mathrm{fb}^{-1}$ & $1 \mathrm{ab}^{-1}$ & $\mathscr{L}_{\text {CLIC }}$ & $100 \mathrm{fb}^{-1}$ & $500 \mathrm{fb}^{-1}$ & $1.5 \mathrm{ab}^{-1}$ & $3 \mathrm{ab}^{-1}$ \\
\hline
\end{tabular}

Table 2: Centre-of-mass energies and expected integrated luminosities of ILC prototypes (left) and CLIC prototypes (right).

beam parameters as given in the ILC Technical Design Report and in the CLIC Conceptual Design Report. Moreover, standard acceptance cuts for a linear collider have been applied to the final-state charged leptons:

$$
E_{\ell}>10 \mathrm{GeV}, \quad\left|\cos \left(\theta_{\ell}\right)\right|<0.95,
$$

where $E_{\ell}$ are the energies of the charged leptons $\left(\ell=e^{ \pm}, \mu^{ \pm}, \tau^{ \pm}\right)$and $\theta_{\ell}$ are their angles with respect to the beam direction. 


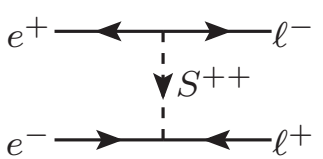

(a)
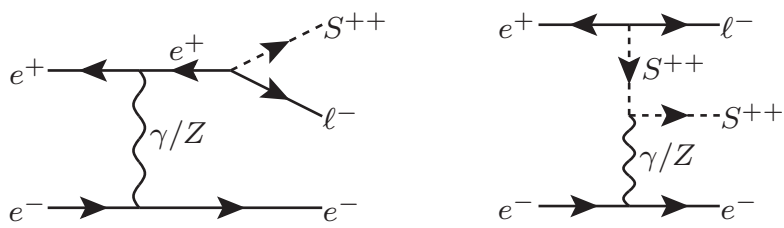

(b)

Figure 3: Feynman diagrams representing: (a) DCS exchange via t-channel in $e^{+} e^{-} \rightarrow \ell^{+} \ell^{-}$processes; $(b)$ contributions to single DCS production at $e^{+} e^{-}$colliders.

The charged lepton pair production at $e^{+} e^{-}$colliders can be sensitive to the couplings $\lambda_{1 j}$ of the DCS to the charged leptons. In this kind of process a DCS is exchanged in the t-channel, enhancing the cross section compared to the SM. The corresponding Feynman diagram is depicted in Figure 3a. Interestingly, when the produced charged leptons have the same flavour, these processes are sensitive to single couplings and give direct bounds on $\lambda_{11}, \lambda_{12}$ or $\lambda_{13}$, complementary to the low-energy bounds, which always constrain a combination of couplings.

In Figure 4 the significance contours for discovery are shown as functions of the mass and the coupling of the DCS involved in the process, both for the ILC and the CLIC, for a muon pair production. As the DCS Eq.1.1 only couples to right-handed leptons, the polarization of the collider beams produces an enhancement of the cross section and consequentely an improvement of the sensitivity. The ILC is designed with the option to polarize the electron beam up to $P_{e^{-}}= \pm 80 \%$ and the positron beam up to $P_{e^{+}}=\mp 30 \%$ [30], while the CLIC will have the option to polarize the electron beam up to $P_{e^{-}}= \pm 80 \%$ [32].
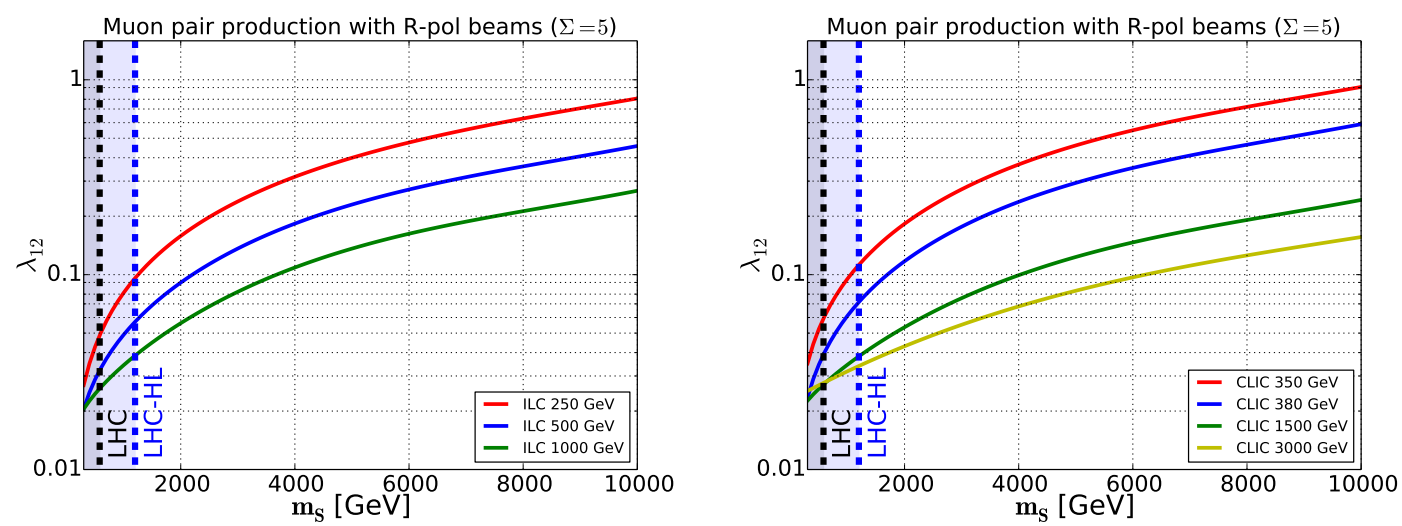

Figure 4: Countours of the cross section of $e^{+} e^{-} \rightarrow \mu^{+} \mu^{-}$with $\Sigma=5$ for different values of the coupling and the mass of the DCS, at ILC with right-polarised beams (left) and CLIC with right-polarised electron beam (right).

To complete the picture, the actual sensitivity of low-energy experiments has to be included in the previous analysis. A combined upper limit on the product $\lambda_{11} \lambda_{12}$ can be extracted from the measurement of the $\mu \rightarrow 3 e$ decay (under the assumption that the other $\lambda_{i j}$ couplings do not lie 
in corners of the parameter space where important cancellation effects take place). The current limit is set to $\mathrm{BR} \leq 10^{-12}$ by the SINDRUM experiment [21] and is expected to be improved by several orders of magnitude by the Mu3e experiment $\left(\mathrm{BR} \leq 5 \cdot 10^{-15}\right.$ in the Phase I) $[35,36]$. Instead, the $e^{+} e^{-}$colliders could explore $\lambda_{11}$ and $\lambda_{12}$ independently. The combined analysis is shown in Figure 5, which highlights the strong complementarity between the low- and high-energy investigations.

The leptonic colliders offer the opportunity to explore a new production channel that is absent at the LHC: the on-shell production of a SU(2)-singlet DCS. The production proceeds via boson fusion and via radiation of the DCS from initial or final leptonic states, in association with two same-sign uncorrelated leptons, when the collider energy is compatible with the mass of the particle. Examples of diagrams are reported in Figure 3b.

In Figure 6 the normalised invariant mass distributions of the electron pairs are displayed for different values of the mass $m_{S}$ and $\Gamma_{S} / m_{S}=5 \%, 10 \%$ at the stage 3 of CLIC with $3 \mathrm{ab}^{-1}$ luminosity and unpolarised electron beam. They are accompanied by the total cross sections, that can be rescaled to account for different values of $\lambda_{11}$. Notice that above the pair-production threshold this production mode dominates and most of the production events contribute to the peak. On the contrary, below the pair-production threshold a shoulder appears beside the peak, in the region of lower invariant masses. Contributions come mainly from the uncorrelated leptons associated to the lepton pair produced by the decay of the DCS, with a subleading contribution from topologies that acquire importance when the DCS is considerably off-shell.
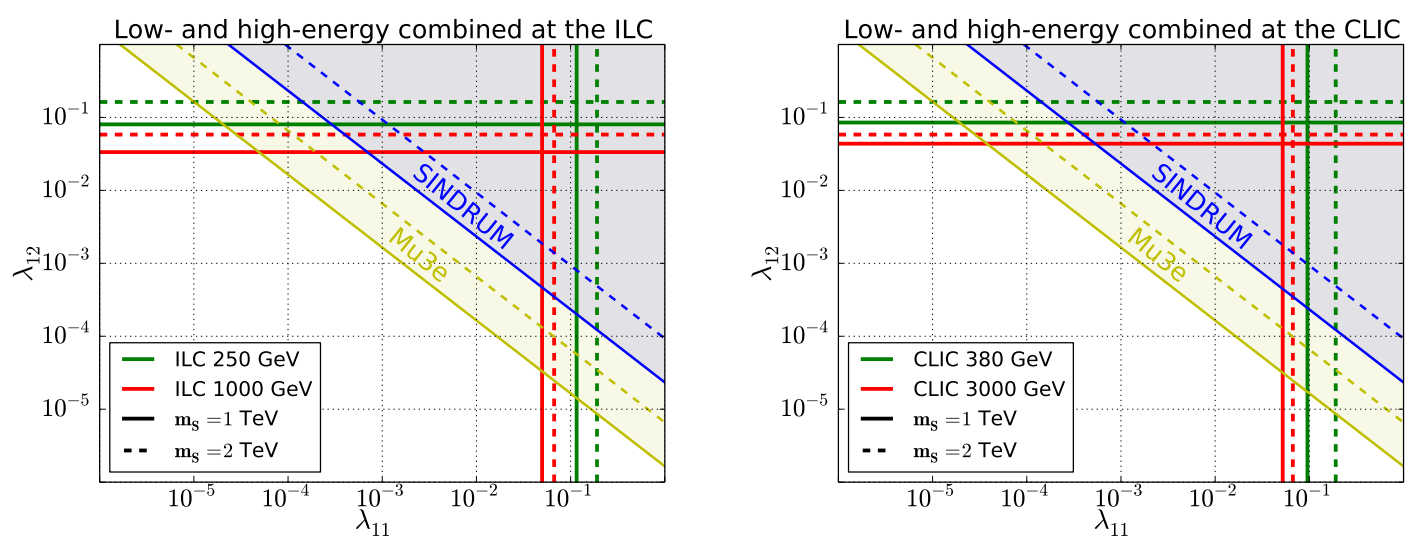

Figure 5: Limits from SINDRUM and discovery power at the ILC (left panel) and CLIC (right panel) prototypes and at the Mu3e experiment (both panels).

\section{Acknowledgments}

These results are selected from the work [15] done in collaboration with Andreas Crivellin, Luca Panizzi, Giovanni Marco Pruna and Adrian Signer. The work of the author has been partially supported by the SNSF contract 200021_160156. 

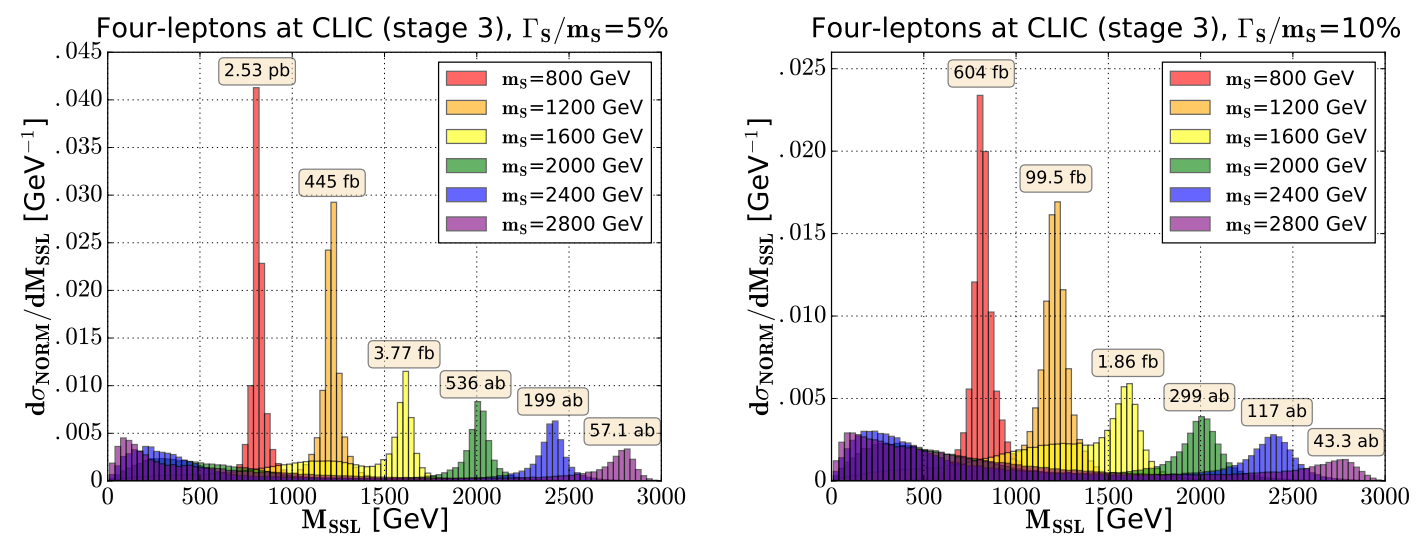

Figure 6: Normalised distributions for different values of the mass of the DCS are shown for $\lambda_{11}=1$ and $\Gamma_{S} / m_{S}=5 \%, 10 \%$ at the stage 3 of CLIC with $3 \mathrm{ab}^{-1}$ luminosity and unpolarised electron beam. The total cross section for each case is reported on the label of the corresponding peak.

\section{References}

[1] J. C. Pati and A. Salam, Lepton Number as the Fourth Color, Phys. Rev. D10 (1974) 275-289.

[2] R. N. Mohapatra and J. C. Pati, A Natural Left-Right Symmetry, Phys. Rev. D11 (1975) 2558.

[3] G. Senjanovic and R. N. Mohapatra, Exact Left-Right Symmetry and Spontaneous Violation of Parity, Phys. Rev. D12 (1975) 1502.

[4] W. Konetschny and W. Kummer, Nonconservation of Total Lepton Number with Scalar Bosons, Phys. Lett. 70B (1977) 433-435.

[5] G. Beall, M. Bander and A. Soni, Constraint on the Mass Scale of a Left-Right Symmetric Electroweak Theory from the K(L) K(S) Mass Difference, Phys. Rev. Lett. 48 (1982) 848.

[6] R. N. Mohapatra and J. D. Vergados, A New Contribution to Neutrinoless Double Beta Decay in Gauge Models, Phys. Rev. Lett. 47 (1981) 1713-1716.

[7] A. Halprin, Neutrinoless Double Beta Decay and Muonium - Anti-Muonium Transitions, Phys. Rev. Lett. 48 (1982) 1313-1316.

[8] T. P. Cheng and L.-F. Li, Neutrino Masses, Mixings and Oscillations in SU(2) $\times U(1)$ Models of Electroweak Interactions, Phys. Rev. D22 (1980) 2860.

[9] M. Magg and C. Wetterich, Neutrino Mass Problem and Gauge Hierarchy, Phys. Lett. 94B (1980) 61-64.

[10] J. Schechter and J. W. F. Valle, Neutrino Masses in SU(2) $\times$ U(1) Theories, Phys. Rev. D22 (1980) 2227.

[11] R. N. Mohapatra and G. Senjanovic, Neutrino Mass and Spontaneous Parity Violation, Phys. Rev. Lett. 44 (1980) 912.

[12] A. Zee, Quantum Numbers of Majorana Neutrino Masses, Nucl. Phys. B264 (1986) 99-110.

[13] K. S. Babu, Model of 'Calculable' Majorana Neutrino Masses, Phys. Lett. B203 (1988) 132-136. 
[14] S. F. King, A. Merle and L. Panizzi, Effective theory of a doubly charged singlet scalar: complementarity of neutrino physics and the LHC, JHEP 11 (2014) 124, [1406.4137].

[15] A. Crivellin, M. Ghezzi, L. Panizzi, G. M. Pruna and A. Signer, Low- and high-energy phenomenology of a doubly charged scalar, 1807.10224.

[16] L. Willmann et al., New bounds from searching for muonium to anti-muonium conversion, Phys. Rev. Lett. 82 (1999) 49-52, [hep-ex/9807011].

[17] Mu3E collaboration, N. Berger, The Mu3e Experiment, Nucl. Phys. Proc. Suppl. $248-250$ (2014) $35-40$.

[18] MU2E collaboration, R. M. Carey et al., Proposal to search for $\mu^{-} N \rightarrow e^{-} N$ with a single event sensitivity below 10 $10^{-16}$, FERMILAB-PROPOSAL-0973, 2008.

[19] COMET collaboration, Y. G. Cui et al., Conceptual design report for experimental search for lepton flavor violating $\mu^{-}-e^{-}$conversion at sensitivity of $10^{-16}$ with a slow-extracted bunched proton beam (COMET), KEK-2009-10, 2009.

[20] MEG II collaboration, A. M. Baldini et al., The design of the MEG II experiment, Eur. Phys. J. C78 (2018) 380, [1801.04688].

[21] SINDRUM collaboration, U. Bellgardt et al., Search for the Decay $\mu^{+} \rightarrow e^{+} e^{+} e^{-}$, Nucl. Phys. B299 (1988) 1-6.

[22] MEG collaboration, A. M. Baldini et al., Search for the lepton flavour violating decay $\mu^{+} \rightarrow \mathrm{e}^{+} \gamma$ with the full dataset of the MEG experiment, Eur. Phys. J. C76 (2016) 434, [1605. 05081].

[23] HFLAV collaboration, Y. Amhis et al., Averages of b-hadron, c-hadron, and $\tau$-lepton properties as of summer 2016, Eur. Phys. J. C77 (2017) 895, [1612.07233].

[24] BABAR collaboration, B. Aubert et al., Searches for Lepton Flavor Violation in the Decays $\tau^{ \pm} \rightarrow e^{ \pm} \gamma$ and $\tau^{ \pm} \rightarrow \mu^{ \pm} \gamma$, Phys. Rev. Lett. 104 (2010) 021802, [0 908.2381$]$.

[25] SINDRUM II collaboration, W. H. Bertl et al., A Search for muon to electron conversion in muonic gold, Eur. Phys. J. C47 (2006) 337-346.

[26] ATLAS collaboration, G. Aad et al., Search for doubly-charged Higgs bosons in like-sign dilepton final states at $\sqrt{s}=7 \mathrm{TeV}$ with the ATLAS detector, Eur. Phys. J. C72 (2012) 2244, [1210 . 5070].

[27] CMS collaboration, S. Chatrchyan et al., A search for a doubly-charged Higgs boson in pp collisions at $\sqrt{s}=7$ TeV, Eur. Phys. J. C72 (2012) 2189, [1207.2666].

[28] ATLAS collaboration, M. Aaboud et al., Search for doubly charged Higgs boson production in multi-lepton final states with the ATLAS detector using protonâAŞproton collisions at $\sqrt{s}=13 \mathrm{TeV}$, Eur. Phys. J. C78 (2018) 199, [1710 . 09748].

[29] CMS collaboration, A search for doubly-charged Higgs boson production in three and four lepton final states at $\sqrt{s}=13 \mathrm{TeV}$, CMS-PAS-HIG-16-036, 2017.

[30] T. Behnke, J. E. Brau, B. Foster, J. Fuster, M. Harrison, J. M. Paterson et al., The International Linear Collider Technical Design Report - Volume 1: Executive Summary, 1306.6327.

[31] H. Baer, T. Barklow, K. Fujii, Y. Gao, A. Hoang, S. Kanemura et al., The International Linear Collider Technical Design Report - Volume 2: Physics, 1306.6352.

[32] M. Aicheler, P. Burrows, M. Draper, T. Garvey, P. Lebrun, K. Peach et al., A Multi-TeV Linear Collider Based on CLIC Technology, CERN-2012-007, 2012. 
[33] L. Linssen, A. Miyamoto, M. Stanitzki and H. Weerts, Physics and Detectors at CLIC: CLIC Conceptual Design Report, 1202.5940.

[34] A. Belyaev, N. D. Christensen and A. Pukhov, CalcHEP 3.4 for collider physics within and beyond the Standard Model, Comput. Phys. Commun. 184 (2013) 1729-1769, [1207 . 6082].

[35] A. Blondel et al., Research Proposal for an Experiment to Search for the Decay $\mu \rightarrow e e e$, 1301.6113.

[36] Mu3E collaboration, A.-K. Perrevoort, Searching for Lepton Flavour Violation with the Mu3e Experiment, PoS NuFact2017 (2017) 105, [1802.09851]. 